\title{
A semi-circle theorem in couple-stress fluid in the presence of rotation
}

\author{
Ajaib S. Banyal* \\ Department of Mathematics, Govt. College Nadaun, Hamirpur, \\ (HP) INDIA 177033.
}

\begin{abstract}
The thermal instability of a couple-stress fluid acted upon by uniform vertical rotation and heated from below is investigated. Following the linearized stability theory and normal mode analysis, the paper through mathematical analysis of the governing equations of couple-stress fluid convection with a uniform vertical rotation, for the case of rigid boundaries shows that the complex growth rate $\sigma$ of oscillatory perturbations, neutral or unstable for all wave numbers, must lie inside a semi-circle
\end{abstract}

$|\sigma|^{2}=T_{A}-\left(\pi^{8} F^{2}\right)$

in the right half of a complex $\sigma$-plane, where $T_{A}$ is the Taylor number and $F$ is the couple-stress parameter, which prescribes the upper limits to the complex growth rate of arbitrary oscillatory motions of growing amplitude in a rotatory couple-stress fluid heated from below. Further, It is established that the existence of oscillatory motions of growing amplitude in the present configuration, depends crucially upon the magnitude of the nondimensional number $\frac{T_{A}}{\left(\pi^{8} F^{2}\right)}$, in the sense so long as $0<\frac{T_{A} P}{\left(\pi^{8} F^{2}\right)} \leq 1$, no such motions are possible, and in particular PES is valid.

Keywords: Thermal convection; Couple-Stress Fluid; Rotation; Complex growth rate; Taylor number.

MSC 2000 No.: 76A05, 76E06, 76E15; 76EO7.

\section{INTRODUCTION}

The thermal instability of a fluid layer with maintained adverse temperature gradient by heating the underside plays an important role in Geophysics, interiors of the Earth, Oceanography and Atmospheric Physics etc. A detailed account of the theoretical and experimental study of the onset of Bénard Convection in Newtonian fluids, under varying assumptions of hydrodynamics and hydromagnetics, has been given by Chandrasekhar [2]. The use of Boussinesq approximation has been made throughout, which states that the density changes are disregarded in all other terms in the equation of motion except the external force term. Sharma et al. [3] has considered the effect of suspended particles on the onset of Bénard convection in hydromagnetics. The fluid has been considered to be Newtonian in all above studies. Scanlon and Segel [4] have considered the effect of

${ }^{*}$ Corresponding author. E-mail: ajaibbanyal@rediffmail.com, Fax No.: 01972232688 
suspended particles on the onset of Bénard convection and found that the critical Rayleigh number was reduced solely because the heat capacity of the pure fluid was supplemented by that of the particles.

With the growing importance of non-Newtonian fluids in modern technology and industries, the investigations on such fluids are desirable. Stokes [5] proposed and postulated the theory of couple-stress fluid. One of the applications of couple-stress fluid is its use to the study of the mechanism of lubrication of synovial joints, which has become the object of scientific research. A human joint is a dynamically loaded bearing which has articular cartilage as the bearing and synovial fluid as lubricant. When fluid film is generated, squeeze film action is capable of providing considerable protection to the cartilage surface. The shoulder, knee, hip and ankle joints are the loaded-bearing synovial joints of human body and these joints have low-friction coefficient and negligible wear. Normal synovial fluid is clear or yellowish and is a viscous, non-Newtonian fluid. According to the theory of Stokes [5], couple-stresses are found to appear in noticeable magnitude in fluids very large molecules. Since the long chain hylauronic acid molecules are found as additives in synovial fluid. Walicki and Walicka [6] modeled synovial fluid as couple-stress fluid in human joints. Sharma and Thakur [7] have studied the thermal convection in couple-stress fluid in porous medium in hydromagnetics. Sharma and Sharma [8] have studied the couple-stress fluid heated from below in porous medium. The use of magnetic field is being made for the clinical purposes in detection and cure of certain diseases with the help of magnetic field devices.

Sharma et al. [9] have studied the effect of suspended particles on couple-stress fluid heated from below and found that suspended particles have stabilizing effect on the system. Sharma and Sharma [10] have studied the effect of suspended particles on couple-stress fluid heated from below in the presence of rotation and magnetic field and found that rotation has a stabilizing effect while dust particles have a destabilizing effect on the system. Sunil et al. [11] have studied the effect of suspended particles on couple-stress fluid heated and soluted from below in porous medium and found that suspended particles have stabilizing effect on the system. Thermosolutal convection in a couple-stress fluid in the presence of magnetic field and rotation, separately, has been investigated by Kumar and Singh [12 \& 13]. Kumar and Kumar [14] have studied the combined effect of dust particles, magnetic field and rotation on couple-stress fluid heated from below and for the case of stationary convection, found that dust particles have destabilizing effect on the system, where as the rotation is found to have stabilizing effect on the system, however couplestress and magnetic field are found to have both stabilizing and destabilizing effects under certain conditions. Sunil et al. [15] have studied the global stability for thermal convection in a couple-stress fluid heated from below and found couple-stress fluids are thermally more stable than the ordinary viscous fluids.

Pellow and Southwell [16] proved the validity of PES for for the classical RayleighBénard convection problem. Banerjee et al. [17] gave a new scheme for combining the governing equations of thermohaline convection, which is shown to lead to the bounds for the complex growth rate of the arbitrary oscillatory perturbations, neutral or unstable for all combinations of dynamically rigid or free boundaries and, Banerjee and Banerjee [18] established a criterion on characterization of non-oscillatory motions in hydrodynamics which was further extended by Gupta et al. [19]. However no such result existed for nonNewtonian fluid configurations, in general and for couple-stress fluid configurations, in particular. Banyal [20] have characterized the non-oscillatory motions in couple-stress fluid in the presence of suspended particles. 
Keeping in mind the importance of non-Newtonian fluids, the present paper is an attempt to prescribe the upper limits to the complex growth rate of arbitrary oscillatory motions of growing amplitude, in a layer of incompressible couple-stress fluid heated from below in the presence of uniform vertical rotation opposite to force field of gravity, when the bounding surfaces of infinite horizontal extension, at the top and bottom of the fluid are rigid.

\section{FORMULATION OF THE PROBLEM AND PERTURBATION EQUATIONS}

Considered an infinite, horizontal, incompressible couple-stress fluid layer, of thickness $d$, heated from below so that, the temperature and density at the bottom surface $z=0$ are $T_{0}, \rho_{0}$ respectively and at the upper surface $z=d$ are $T_{d}, \rho_{d}$ and that a uniform adverse temperature gradient $\beta\left(=\left|\frac{d T}{d z}\right|\right)$ is maintained. The fluid is acted upon by a uniform vertical rotation $\vec{\Omega}(0,0, \Omega)$. Let $\rho, p, T$ and $\vec{q}(u, v, w)$ denote respectively the density, pressure, temperature and velocity of the fluid. Then the momentum balance, mass balance equations of the couplestress fluid (Stokes [5]; Chandrasekhar [2] and Scanlon and Segel [4]) are

$$
\begin{aligned}
& \frac{\partial \vec{q}}{\partial t}+(\vec{q} \cdot \nabla) \vec{q}=-\frac{1}{\rho_{0}} \nabla p+\vec{g}\left(1+\frac{\delta \rho}{\rho_{0}}\right)+\left(v-\frac{\mu^{\prime}}{\rho_{0}} \nabla^{2}\right) \nabla^{2} \vec{q}+2(\vec{q} \times \vec{\Omega}), \\
& \nabla . \vec{q}=0,
\end{aligned}
$$

The equation of state

$$
\rho=\rho_{0}\left[1-\alpha\left(T-T_{0}\right)\right]
$$

Where the suffix zero refer to the values at the reference level $z=0$. Here $\vec{g}(0,0,-g)$ is acceleration due to gravity.

Let $c_{v}$ denote the heat capacity of the fluid at constant volume. Assuming that the particles and the fluid are in thermal equilibrium, the equation of heat conduction gives

$$
\rho_{0} c_{v}\left(\frac{\partial}{\partial t}+\vec{q} . \nabla\right) T=\vec{q} \nabla^{2} T,
$$

Or

$$
\frac{\partial T}{\partial t}+(\vec{q} \cdot \nabla) T=\kappa \nabla^{2} T
$$

The kinematic viscosity $v$, couple-stress viscosity $\mu^{\prime}$, thermal diffusivity $\kappa=\frac{q}{\rho_{0} c_{v}}$, and
, efficient of thermal expansion $\alpha$ are all assumed to be constants.

The basic motionless solution is

$$
\vec{q}=(0,0,0), T=T_{0}-\beta z, \vec{\Omega}=(0,0, \Omega) \text { and } \rho=\rho_{0}(1+\alpha \beta z)
$$


Assume small perturbations around the basic solution and let $\delta \rho, \delta p, \theta$ and $\vec{q}(u, v, w)$ denote respectively the perturbations in density, pressure $p$, temperature $T$ and couple-stress fluid velocity $(0,0,0)$. The change in density $\delta \rho$ caused mainly by the perturbation $\theta$ in temperature is given by

$$
\delta \rho=-\alpha \rho_{0} \theta
$$

Then the linearized perturbation equations of the couple-stress fluid becomes

$$
\begin{aligned}
& \frac{\partial \vec{q}}{\partial t}=-\frac{1}{\rho_{0}} \nabla \delta p-\vec{g} \alpha \theta+\left(v-\frac{\mu^{\prime}}{\rho_{0}} \nabla^{2}\right) \nabla^{2} \vec{q}+2(\vec{q} \times \vec{\Omega}), \\
& \nabla \cdot \vec{q}=0 \\
& \frac{\partial \theta}{\partial t}=\beta w+\kappa \nabla^{2} \theta
\end{aligned}
$$

Within the framework of Boussinesq approximation, equations (9), (10), (11) and (12) give

$$
\begin{aligned}
& {\left[\frac{\partial}{\partial t} \nabla^{2} w-g \alpha\left(\frac{\partial^{2} \theta}{\partial x^{2}}+\frac{\partial^{2} \theta}{\partial y^{2}}\right)+2 \Omega \frac{\partial \varsigma}{\partial z}\right]=\left(v-\frac{\mu^{\prime}}{\rho_{0}} \nabla^{2}\right) \nabla^{4} w,} \\
& {\left[\frac{\partial \varsigma}{\partial t}-2 \Omega \frac{\partial w}{\partial z}\right]=\left(v-\frac{\mu^{\prime}}{\rho_{0}} \nabla^{2}\right) \nabla^{2} \varsigma,}
\end{aligned}
$$

Together with (19), where $\nabla^{2}=\frac{\partial^{2}}{\partial x^{2}}+\frac{\partial^{2}}{\partial y^{2}}+\frac{\partial^{2}}{\partial z^{2}}$ and $\varsigma=\frac{\partial v}{\partial x}-\frac{\partial u}{\partial y}$ denote the $z$-component
vorticity.

\section{NORMAL MODE ANALYSIS}

Analyzing the disturbances into normal modes, we assume that the Perturbation quantities are of the form

$$
[w, \theta, \varsigma]=[W(z), \Theta(z), Z(z)] \operatorname{Exp}\left(i k_{x} x+i k_{y} y+n t\right)
$$

Where $k_{x}, k_{y}$ are the wave numbers along the $x$ and $y$-directions respectively $k=\left(k_{x}^{2}+k_{y}^{2}\right)^{\frac{1}{2}}$, is the resultant wave number and $n$ is the growth rate which is, in general, a complex constant. 
Using (12), equations (9), (10) and (11), on using (8), in non-dimensional form, become

$$
\begin{aligned}
& \left(D^{2}-a^{2}\right)\left[\sigma+F\left(D^{2}-a^{2}\right)^{2}-\left(D^{2}-a^{2}\right)\right] W=-\frac{g \alpha d^{2} a^{2} \Theta}{v}-\sqrt{T_{A}} d D Z, \\
& {\left[\left\{1-F\left(D^{2}-a^{2}\right)\right\}\left(D^{2}-a^{2}\right)-\sigma\right] Z=-\frac{\sqrt{T}_{A}}{d} D W,} \\
& \left(D^{2}-a^{2}-p_{1} \sigma\right) \Theta=-\frac{\beta d^{2}}{\kappa} W,
\end{aligned}
$$

where

$a=k d, \sigma=\frac{n d^{2}}{v}, p_{1}=\frac{v}{\kappa}, F=\frac{\mu^{\prime}}{\rho_{0} d^{2} v}, T_{A}=\frac{4 \Omega^{2} d^{4}}{v^{2}} D=\frac{d}{d z}$ and $D_{\oplus}=d D$ and dropping $(\oplus)$ for convenience. Here $p_{1}=\frac{v}{\kappa}$, is the thermal prandtl number, $F$ is the couple-stress parameter and $T_{A}$ is the Taylor number.

Substituting $W=W_{\oplus}, \Theta=\frac{\beta d^{2}}{\kappa} \Theta_{\oplus}$, and $Z=\frac{\sqrt{T}_{A}}{d} Z_{\oplus}$ in equations (13), (14) and (15) and dropping $(\oplus)$ for convenience, in non-dimensional form becomes,

$$
\begin{aligned}
& \left(D^{2}-a^{2}\right)\left[\sigma+F\left(D^{2}-a^{2}\right)^{2}-\left(D^{2}-a^{2}\right)\right] W=-R a^{2} \Theta-T_{A} D Z, \\
& {\left[\left\{1-F\left(D^{2}-a^{2}\right)\right\}\left(D^{2}-a^{2}\right)-\sigma\right] Z=-D W} \\
& \left(D^{2}-a^{2}-p_{1} \sigma\right) \Theta=-W
\end{aligned}
$$

Where $R=\frac{g \alpha \beta d^{4}}{\kappa \nu}$, is the thermal Rayleigh number.

Since both the boundaries rigid and are maintained at constant temperature, the perturbations in the temperature are zero at the boundaries. The appropriate boundary conditions with respect to which equations (16), (17) and (18) must be solved are

$$
W=D W=0, \Theta=0 \text { and } Z=0 \text { at } z=0 \text { and } z=1 .
$$

Equations (16)-(18), along with boundary conditions (19), pose an eigenvalue problem for $\sigma$ and we wish to Characterize $\sigma_{i}$ when $\sigma_{r} \geq 0$. 


\section{MATHEMATICAL ANALYSIS}

We prove the following theorems:

Theorem: If $R>0, F>0, T_{A}>0, \sigma_{r} \geq 0$ and $\sigma_{i} \neq 0$ then the necessary condition for the existence of non-trivial solution $(W, \Theta, Z)$ of equations (16), (17) and (18) together with boundary conditions (19) is that

$$
|\sigma|^{2}\left\langle T_{A}-\left(\pi^{8} F^{2}\right)\right.
$$

Proof: Multiplying equation (16) by $W^{*}$ (the complex conjugate of $W$ ) throughout and integrating the resulting equation over the vertical range of $z$, we get

$$
\begin{aligned}
& \sigma \int_{0} W^{*}\left(D^{2}-a^{2}\right) W d z+F \int_{0}^{1} W^{*}\left(D^{2}-a^{2}\right)^{3} W d z \\
& -\int_{0}^{1} W^{*}\left(D^{2}-a^{2}\right)^{2} W d z=-R a^{2} \int_{0}^{1} W^{*} \Theta d z-T_{A} \int_{0}^{1} W^{*} D Z d z,
\end{aligned}
$$

Taking complex conjugate on both sides of equation (18), we get

$$
\left(D^{2}-a^{2}-p_{1} \sigma^{*}\right) \Theta^{*}=-W^{*}
$$

Therefore, using (21), we get

$$
\int_{0}^{1} W^{*} \Theta d z=-\int_{0}^{1} \Theta\left(D^{2}-a^{2}-p_{1} \sigma^{*}\right) \Theta^{*} d z
$$

Also taking complex conjugate on both sides of equation (17), we get

$$
\left[\left\{1-F\left(D^{2}-a^{2}\right)\right\}\left(D^{2}-a^{2}\right)-\sigma^{*}\right] Z^{*}=-D W^{*},
$$

Therefore, using (23), we get

$$
\int_{0}^{1} W^{*} D Z d z=-\int_{0}^{1} D W^{*} Z d z=\int_{0}^{1} Z\left\{\left(D^{2}-a^{2}\right)-F\left(D^{2}-a^{2}\right)^{2}-\sigma^{*}\right\} Z^{*} d z
$$


Substituting (22) and (24) in the right hand side of equation (20), we get

$$
\begin{aligned}
& \sigma \int_{0}^{1} W^{*}\left(D^{2}-a^{2}\right) W d z+F \int_{0}^{1} W^{*}\left(D^{2}-a^{2}\right)^{3} W d z-\int_{0}^{1} W^{*}\left(D^{2}-a^{2}\right)^{2} W d z \\
& =R a^{2} \int_{0}^{1} \Theta\left(D^{2}-a^{2}-p_{1} \sigma^{*}\right) \Theta^{*} d z-T_{A} \int_{0}^{1} Z\left\{\left(D^{2}-a^{2}\right)-F\left(D^{2}-a^{2}\right)^{2}-\sigma^{*}\right\} Z^{*} d z,
\end{aligned}
$$

Integrating the terms on both sides of equation (25) for an appropriate number of times by making use of the appropriate boundary conditions (19), along with (17), we get

$$
\begin{aligned}
& \sigma \int_{0}^{1}\left\{|D W|^{2}+a^{2}|W|^{2}\right\} d z+F \int_{0}^{1}\left\{\left|D^{3} W\right|^{2}+3 a^{2}\left|D^{2} W\right|^{2}+3 a^{4}|D W|^{2}+a^{6}|W|^{2}\right\} d z \\
& +\int_{0}^{1}\left\{\left|D^{2} W\right|^{2}+2 a^{2}|D W|^{2}+a^{4}|W|^{2}\right\} d z=R a^{2} \int_{0}^{1}\left\{|D \Theta|^{2}+a^{2}|\Theta|^{2}+p_{1} \sigma^{*}|\Theta|^{2}\right\} d z \\
& -T_{A} \int_{0}^{1}\left\{|D Z|^{2}+a^{2}|Z|^{2}\right\} d z-T_{A} F \int_{0}^{1}\left\{\left|D^{2} Z\right|^{2}+2 a^{2}|D Z|^{2}+a^{4}|Z|^{2}\right\} d z-T_{A} \sigma^{*} \int_{0}^{1}|Z|^{2} d z,
\end{aligned}
$$

And equating imaginary parts on both sides of equation (26), and cancelling $\sigma_{i}(0)$ throughout from imaginary part, we get

$$
\int_{0}^{1}\left\{|D W|^{2}+a^{2}|W|^{2}\right\} d z+R a^{2} p_{1} \int_{0}^{1}|\Theta|^{2} d z=T_{A} \int_{0}^{1}|Z|^{2} d z,
$$

We first note that since $W$ and $Z$ satisfy $W(0)=0=W(1)$ and $Z(0)=0=Z(1)$ in addition to satisfying to governing equations and hence we have from the Rayleigh-Ritz inequality [21]

$$
\int_{0}^{1}|D W|^{2} d z \geq \pi^{2} \int_{0}^{1}|W|^{2} d z
$$

and

$$
\int_{0}^{1}|D Z|^{2} d z \geq \pi^{2} \int_{0}^{1}|Z|^{2} d z
$$


Further, for $W(0)=0=W(1)$ and $Z(0)=0=Z(1)$, Banerjee et al. [22] have shown that

$$
\int_{0}^{1}\left|D^{2} W\right|^{2} d z \geq \pi^{2} \int_{0}^{1}|D W|^{2} d z \text { and } \int_{0}^{1}\left|D^{2} Z\right|^{2} d z \geq \pi^{2} \int_{0}^{1}|D Z|^{2} d z
$$

Further, multiplying equation (17) and its complex conjugate (23), and integrating by parts each term on both sides of the resulting equation for an appropriate number of times and making use of boundary condition on $Z$ namely $Z(0)=0=Z(1)$ along with (17), we get

$$
\begin{aligned}
& \int_{0}^{1}\left\{\left|D^{2} Z\right|^{2}+2 a^{2}|D Z|^{2}+a^{4}|Z|^{2}\right\} d z+F^{2} \int_{0}^{1}\left\{\left|D^{4} Z\right|^{2}+4 a^{2}\left|D^{3} Z\right|^{2}+6 a^{4} \int_{0}^{1}\left|D^{2} Z\right|^{2}\right. \\
& \left.+4 a^{6} \int_{0}^{1}|D Z|^{2}+a^{8}|Z|^{2}\right\} d z+2 F \int_{0}^{1}\left\{\left|D^{3} Z\right|^{2}+3 a^{2}\left|D^{2} Z\right|^{2}+3 a^{4} \int_{0}^{1}|D Z|^{2}+a^{6}|Z|^{2}\right\} d z \\
& +2 \sigma_{r} \int_{0}^{1}\left\{|D Z|^{2}+a^{2}|Z|^{2}\right\} d z+2 \sigma_{r} F \int_{0}^{1}\left\{\left|D^{4} Z\right|^{2}+2 a^{2}\left|D^{2} Z\right|^{2}+a^{4}|Z|^{2}\right\} d z \\
& +|\sigma|^{2} \int_{0}^{1}|Z|^{2} d z=\int_{0}^{1}|D W|^{2} d z,
\end{aligned}
$$

Further, by utilizing boundary conditions (19) and equation (17), it follows that

$$
\begin{aligned}
& \int_{0}^{1}\left|D^{2} Z\right|^{2} d z=\text { Real part of }\left[-\int_{0}^{1} D Z^{*} D^{3} Z d z\right] \\
& \leq\left|-\int_{0}^{1} D Z^{*} D^{3} Z d z\right| \\
& \leq\left|\int_{0}^{1} D Z^{*} D^{3} Z d z\right| \\
& \leq \int_{0}^{1}\left|D Z^{*} D^{3} Z\right| d z \\
& \leq \int_{0}^{1}\left|D Z^{*}\right|\left|D^{3} Z\right| d z
\end{aligned}
$$




$$
\begin{aligned}
& \leq \int_{0}^{1}|D Z|\left|D^{3} Z\right| d z \\
& \leq\left[\int_{0}^{1}|D Z|^{2} d z\right]^{\frac{1}{2}}\left[\int_{0}^{1}\left|D^{3} Z\right|^{2} d z\right]^{\frac{1}{2}},
\end{aligned}
$$

(Utilizing Cauchy- Schwartz-inequality),

$$
\leq \frac{1}{\pi}\left[\int_{0}^{1}\left|D^{2} Z\right|^{2} d z\right]^{\frac{1}{2}}\left[\int_{0}^{1}\left|D^{3} Z\right|^{2} d z\right]^{\frac{1}{2}}
$$

(Utilizing inequality (30)),

So that we have

$$
\left[\int_{0}^{1}\left|D^{2} Z\right|^{2} d z\right]^{\frac{1}{2}} \leq \frac{1}{\pi}\left[\int_{0}^{1}\left|D^{3} Z\right|^{2} d z\right]^{\frac{1}{2}}
$$

Which yields

$$
\int_{0}^{1}\left|D^{3} Z\right|^{2} d z \geq \pi^{2} \int_{0}^{1}\left|D^{2} Z\right|^{2} d z
$$

Using inequality (29) and (30), inequality (32) becomes

$$
\int_{0}^{1}\left|D^{3} Z\right|^{2} d z \geq \pi^{6} \int_{0}^{1}|Z|^{2} d z
$$

Also,

$$
\int_{0}^{1}\left|D^{3} Z\right|^{2} d z=\text { Real part of }\left[-\int_{0}^{1} D^{2} Z^{*} D^{4} Z d z\right]
$$


$\leq\left|-\int_{0}^{1} D^{2} Z^{*} D^{4} Z d z\right|$

$\leq\left|\int_{0}^{1} D^{2} Z^{*} D^{4} Z d z\right|$

$\leq \int_{0}^{1}\left|D^{2} Z^{*} D^{4} Z\right| d z$

$\leq \int_{0}^{1}\left|D^{2} Z^{*}\right|\left|D^{4} Z\right| d z$

$\leq \int_{0}^{1}\left|D^{2} Z\right|\left|D^{4} Z\right| d z$

$\leq\left[\int_{0}^{1}\left|D^{2} Z\right|^{2} d z\right]^{\frac{1}{2}}\left[\int_{0}^{1}\left|D^{4} Z\right|^{2} d z\right]^{\frac{1}{2}}$,

(Utilizing Cauchy- Schwartz-inequality),

$$
\leq \frac{1}{\pi}\left[\int_{0}^{1}\left|D^{3} Z\right|^{2} d z\right]^{\frac{1}{2}}\left[\int_{0}^{1}\left|D^{4} Z\right|^{2} d z\right]^{\frac{1}{2}}
$$

(Utilizing inequality (32)),

So that we have

$$
\left[\int_{0}^{1}\left|D^{3} Z\right|^{2} d z\right]^{\frac{1}{2}} \leq \frac{1}{\pi}\left[\int_{0}^{1}\left|D^{4} Z\right|^{2} d z\right]^{\frac{1}{2}},
$$

Which yields

$$
\int_{0}^{1}\left|D^{4} Z\right|^{2} d z \geq \pi^{2} \int_{0}^{1}\left|D^{3} Z\right|^{2} d z
$$


Using inequality (33), inequality (34) becomes

$$
\int_{0}^{1}\left|D^{4} Z\right|^{2} d z \geq \pi^{8} \int_{0}^{1}|Z|^{2} d z
$$

Now $F>0$ and $\sigma_{r} \geq 0$, therefore the equation (31) gives,

$$
F^{2} \int_{0}^{1}\left|D^{4} Z\right|^{2} d z+|\sigma|^{2} \int_{0}^{1}|Z|^{2} d z\left\langle\int_{0}^{1}|D W|^{2} d z\right.
$$

And on utilizing the inequalities (29), (30), (33) and (35), inequality (36) gives

$$
\int_{0}^{1}|z|^{2} d z\left\langle\frac{1}{\left(\pi^{8} F^{2}+|\sigma|^{2}\right)} \int_{0}^{1}|D W|^{2} d z\right.
$$

Now $R>0$ and $T_{A}>0$, utilizing the inequalities (37), the equation (27) gives,

$$
\left[1-\frac{T_{A}}{\left(\pi^{8} F^{2}+|\sigma|^{2}\right)}\right] \int_{0}^{1}|D W|^{2} d z+a^{2} \int_{0}^{1}|W|^{2} d z+R a^{2} p_{1} \int_{0}^{1}|\Theta|^{2} d z\langle 0
$$

and therefore, we must have

$$
|\sigma|^{2}\left\langle T_{A}-\left(\pi^{8} F^{2}\right)\right.
$$

Hence, if

$$
\sigma_{r} \geq 0 \text { and } \sigma_{i} \neq 0, \text { then }|\sigma|^{2}\left\langle T_{A}-\left(\pi^{8} F^{2}\right)\right.
$$

And this completes the proof of the theorem.

In the context of existence of instability in 'oscillatory modes' and that of 'overstability' in the present configuration, we can state prove a theorem as follow:-

Theorem 2: The necessary condition for the existence of instability in 'oscillatory modes' and that of 'overstability' in a couple-stress fluid heated from below, in the presence of uniform vertical rotation is that the Taylor number $T_{A}$ and the couple-stress parameter of the fluid $F$, must satisfy the inequality $\left.\frac{T_{A}}{\pi^{8} F^{2}}\right\rangle 1$, when both the bounding surfaces are rigid 
Proof: The inequality (39) for $\sigma_{r} \geq 0$ and $\sigma_{i} \neq 0$, can be written as

$$
\sigma_{r}^{2}+\sigma_{i}^{2}\left\langle\left[T_{A}-\pi^{8} F^{2}\right]\right.
$$

we necessarily have,

$$
\left.\frac{T_{A}}{\pi^{8} F^{2}}\right\rangle 1
$$

which completes the proof.

Presented otherwise from the point of view of existence of instability as stationary convection, the above theorem can be put in the form as follow:-

Theorem 3: The sufficient condition for the validity of the 'exchange principle' and the onset of instability as a non-oscillatory motions of non-growing amplitude in a couple-stress fluid heated from below, in the presence of uniform vertical rotation is that, $\frac{T_{A}}{\pi^{8} F^{2}} \leq 1$, where $T_{A}$ is the Taylor number and $F$ is the couple-stress parameter, when both the bounding surface are rigid.

or

The onset of instability in a couple-stress fluid heated from below, in the presence of uniform vertical rotation, cannot manifest itself as oscillatory motions of growing amplitude if the Taylor number $T_{A}$ and the couple-stress parameter $F$, satisfy the inequality $\frac{T_{A}}{\pi^{8} F^{2}} \leq 1$, when
both the bounding surfaces are rigid.

\section{CONCLUSIONS}

The inequality (39) for $\sigma_{r} \geq 0$ and $\sigma_{i} \neq 0$, can be written as

$$
\sigma_{r}^{2}+\sigma_{i}^{2}\left\langle\left[T_{A}-\pi^{8} F^{2}\right]\right.
$$

The essential content of the theorem, from the point of view of linear stability theory is that for the configuration of couple-stress fluid of infinite horizontal extension heated form below, having top and bottom bounding surfaces rigid, in the presence of uniform vertical rotation parallel to the force field of gravity, the complex growth rate of an arbitrary oscillatory motions of growing amplitude, must lie inside a semi-circle in the right half of the $\sigma_{r} \sigma_{i}$ - plane whose centre is at the origin and radius is $\sqrt{T_{A}-\pi^{8} F^{2}}$, where $T_{A}$ is the Taylor number and $F$ is the couple-stress parameter, it improved the domain of the result of Banyal and Khanna [1] significantly.

Further, it follows from inequality (39) that a sufficient condition for the validity of the 'principle of exchange of stabilities' in rotatory couple-stress fluid convection is that $\frac{T_{A}}{\pi^{8} F^{2}} \leq 1$. It is therefore clear that the existence of oscillatory motions of growing amplitude in the present configuration, depends crucially upon the magnitude of the non-dimensional 
number $\frac{T_{A}}{\pi^{8} F^{2}}$, in the sense so long as $0<\frac{T_{A}}{\pi^{8} F^{2}} \leq 1,6$ no such motions are possible, and in particular PES is valid.

\section{REFERENCES}

[1] Banyal, A. S. and Khanna, M., (2012), Bounds for Growth Rate of Perturbations in Couple-Stress Fluid in the Presence of Rotation, Global J. of Pure and Appld. Scis. \& Tech., Vol. 2, Issue 2, pp. 24-31.

[2] Chandrasekhar, S. (1981). Hydrodynamic and Hydromagnetic Stability, Dover Publication,

[3] Sharma, R.C., Prakash, K. and Dube, S.N. (1976). Effect of suspended particles on the onset of Bénard convection in hydromagnetics, J. Math. Anal. Appl., USA, Vol. 60 pp. 227-35.

[4] Scanlon, J.W. and Segel, L.A. (1973). Some effects of suspended particles on the onset of Bénard convection, Phys. Fluids. Vol. 16, pp. 1573-78.

[5] Stokes, V.K. (1966). Couple-stress in fluids, Phys. Fluids, Vol. 9, pp. 1709-15.

[6] Walicki, E. and Walicka, A. (1999). Inertial effect in the squeeze film of couple-stress fluids in biological bearings, Int. J. Appl. Mech. Engg., Vol. 4, pp. 363-73.

[7] Sharma, R.C. and Thakur, K. D. (2000). Couple stress-fluids heated from below in hydromagnetics, Czech. J. Phys., Vol. 50, pp. 753-58.

[8] Sharma, R.C. and Sharma S. (2001). On couple-stress fluid heated from below in porous medium, Indian J. Phys, Vol. 75B, pp. 59-61.

[9] Sharma, R.C., Sunil, Sharma, Y. D. and Chandel, R.S. (2002). On couple-stress fluid permeated with suspended particles heated from below, Archives of Mechanics, 54(4) pp. 287-298.

[10] Sharma, R.C. and Sharma, M. (2004). Effect of suspended particles on couple-stress fluid heated from below in the presence of rotation and magnetic field, Indian J. pure. Appl. Math., Vol. 35(8), pp. 973-989.

[11] Sunil, Sharma, R.C. and Chandel, R.S. (2004). Effect of suspended particles on couple-stress fluid heated and soluted from below in porous medium, J. of Porous Media, Vol. 7, No. 1 pp. 9-18.

[12] Kumar, P. and Singh, M. (2008), Magneto thermosolutal convection in a couple-stress fluid, Ganita Sandesh (india), Vol. 21(2).

[13] Singh, M. and. Kumar, P. (2009), Rotatory thermosolutal convection in a couple-stress fluid, Z. Naturforsch, 64a, 7(2009).

[14] Kumar, V. and Kumar, S. (2011). On a couple-stress fluid heated from below in hydromagnetics, Appl. Appl. Math., Vol. 05(10), pp. 1529-1542.

[15] Sunil, Devi, R. and Mahajan, A. (2011), Global stability for thermal convection in a couple stressfluid, Int. comm.. Heat and Mass Transfer, 38, pp. 938-942.

[16] Pellow, A., and Southwell, R.V. (1940). On the maintained convective motion in a fluid from below. Proc. Roy. Soc. London A 176, 312-43.

[17] Banerjee, M.B., Katoch, D.C., Dube,G.S. and Banerjee, K. (1981). Bounds for growth rate of perturbation in thermohaline convection. Proc. R. Soc. A378, 301-04.

[18] Banerjee, M. B., and Banerjee, B. (1984). A characterization of nonoscillatory motions in magnetohydronamics. Ind. J. Pure \& Appl Maths., 15(4), 377-382.

[19] Gupta, J.R., Sood, S.K., and Bhardwaj, U.D. (1986). On the characterization of nonoscillatory motions in rotatory hydromagnetic thermohaline convection, Indian J. pure appl. Math., 17(1) pp. 100-107. 
[20] Banyal, A.S., (2011) A characterization of non-oscillatory motions in couple-stress fluid in the presence of suspended particles (2011), J. Comp. and Math. Scis. (JCMS), Vol. 2 ( 3), pp. 537-545.

[21] Schultz, M.H. (1973). Spline Analysis, Prentice Hall, Englewood Cliffs, New Jersy.

[22] Banerjee, M.B., Gupta, J.R. and Prakash, J. (1992). On thermohaline convection of Veronis type, J. Math. Anal. Appl., Vol. 179, No. 2 pp. 327-334. 